\title{
First Outbreak of Occupational Brucellosis Involving Multiple Clusters - Hubei Province, China, 2019
}

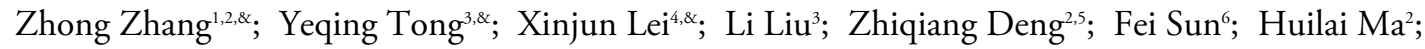 \\ Zhaorui Chang'; Lijie Zhang'; Huihui Liu'; Qi Chen³; Jianzhong Zheng'; Haibing Chen'; \\ Ximei Sun'; Yang Wu ${ }^{3, * *}$; Xuhua Guan ${ }^{3, *}$
}

\section{Summary \\ What is already known on this topic? \\ Human brucellosis, a neglected zoonotic disease, causes more than 500,000 new cases each year globally. The disease is of major public health concern in China, and northern provinces are traditionally endemic areas. \\ What is added by this report? \\ This is the first published outbreak of occupational brucellosis involving multiple clusters in Hubei Province. This investigation characterizes the transmission chain of the outbreak and reveals that provinces south of the Yangtze River are faced with a series of challenges and hurdles to overcome including, but not limited to, health education, law enforcement, and occupational protection. \\ What are the implications for public health practice? \\ In addition to measures aimed at this outbreak, the local agricultural department has issued special notices based on this investigation to adjust and strengthen local eradication program of brucellosis.}

The incidence of human brucellosis is surging dramatically in southern provinces of China (1-3), including provincial-level administrative divisions (PLADs) along and to the south of the Yangtze River*, and no brucellosis outbreaks were reported in southern Hubei Province before this investigation (4-5). An eradication program of brucellosis has been put into action in Hubei since 2017.

On June 3, 2019, 2 clusters of brucellosis involving 5 cases in Xianning, a prefecture-level city in southern Hubei, were reported to the National Public Health Emergency Reporting Information System (NPHERIS), and 1 cluster involved 3 employees of $\mathrm{L}$ mutton restaurant in Chongyang County, Xianning with the other involving 2 goat keepers of J goat farm in Jiayu County, Xianning. A field investigation was initiated to identify the infection source and risk factors.

\section{INVESTIGATION AND RESULTS}

Suspected cases were defined as residents of Chongyang County and suppliers of the $\mathrm{L}$ mutton restaurant who developed two or more of the following symptoms from January 1 to June 31, 2019: fever ( $\geq$ $37.5{ }^{\circ} \mathrm{C}$ ), fatigue, night sweats, and joint pain excluding patients with confirmed diagnosis for other diseases. Confirmed cases were defined as suspected cases with positive $1: 100(++)$ or above serum agglutination test (SAT) for Brucella.

Case finding was carried out by interviewing cases, doctors, restaurant managers, and others employees, reviewing Infectious Disease Reporting Management Information System and medical records, and implementing symptom surveillance in local major hospitals.

Serum samples of all suspect cases were collected and tested. If the Rose Bengal plate agglutination test (RBPT) or SAT was positive, detailed demographic information, clinical information, eating habits, occupational history, preventive measures, and sources of animal products were collected. A field investigation was also initiated to ascertain possible polluted environments which these patients were exposed to.

A total of 8 cases (including 5 initial cases mentioned above) of brucellosis were identified with 6 patients experiencing onset of illness in April and 2 patients in May. All cases were confirmed. The most commonly reported symptoms were fever $(88 \%)$, joint pain $(75 \%)$, and fatigue $(75 \%)$, and they were all

\footnotetext{
* These include Jiangsu, Anhui, Shanghai, Hubei, Sichuan, Zhejiang, Chongqing, Jiangxi, Hunan, Guizhou, Fujian, Yunnan, Guangdong, Guangxi, and Hainan of the mainland of China.
} 
discharged after a treatment of doxycycline and rifampin (42 d). Of these patients, 6 came from Chongyang County, and 2 from Jiayu County, and their ages ranged from 26 to 57 years old with a median age of 49 years. Their suspected exposures included goat-slaughtering, abortive ewes, and environmental exposure. Time intervals between onset of symptoms and diagnosis varied from 6 to 43 days, and the onset-to-diagnosis intervals of 6 cases exceeded 1 month.

The trade relationship among the work sites was further investigated. In Hubei, mutton consumption peaks between October and February of the next year, and $\mathrm{L}$ mutton restaurant mainly engaged in mutton hot pots and mutton noodles. During the off-season, the restaurant bought frozen mutton from B wholesale market in Wuhan, whose mutton originated from a market in Huanghua, Hebei Province of Northern China. During the peak months, it bought live goats from J goat farm and then slaughtered them in a back room of the restaurant. J goat farm bought live goats from M live goat market in Fang County, Shiyan City, northern Hubei Province (Figure 1).

During the investigation, a total of 19 serum samples were collected and 8 of them tested positive for RBPT and SAT. A strain of Brucella melitensis was isolated (Table 1).

Given that most cases had a suspected exposure to goat-slaughtering, we hypothesized that slaughtering was a key risk factor of infection. Subsequently, we investigated the exposure histories of 21 individuals, including all employees of $\mathrm{L}$ mutton restaurant, $\mathrm{J}$ goat farm, and D butcher's. No employees wore any personal protective equipment at work, and no patients had a history of consuming unpasteurized contaminated animal products or a history of travelling to endemic areas beforehand. The results showed that slaughtering without protective measures was a risk factor $(\mathrm{RR}=11.38,95 \% \mathrm{CI}: 1.70-76.14)$ (Table 2), and 6 of the 8 patients had not heard of brucellosis before and no patients had an awareness of clinical signs and symptoms of human brucellosis.

Further field investigation revealed that the slaughterhouse of $\mathrm{L}$ mutton restaurant was a low-

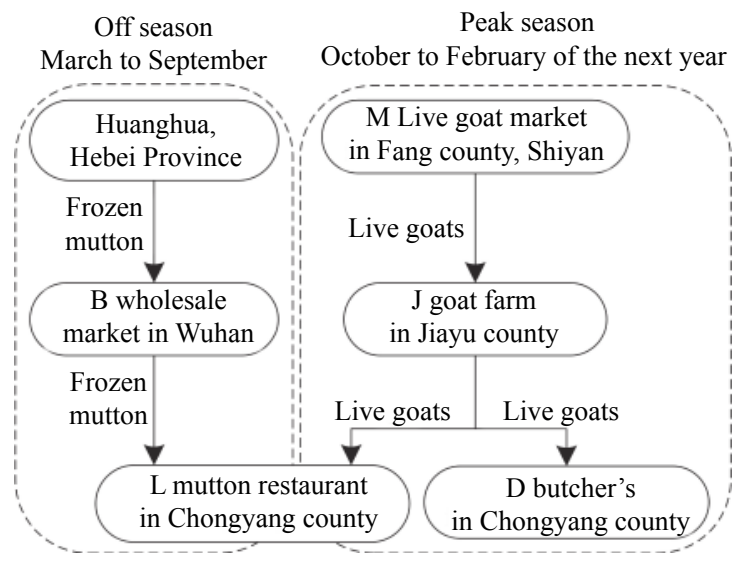

FIGURE 1. Transaction flow charts of an outbreak of brucellosis in Hubei Province, China, 2019.

TABLE 1. Laboratory results of an outbreak of brucellosis in Hubei Province, China, 2019.

\begin{tabular}{ccccc}
\hline Source of samples & Samples collected & RBPT positive & SAT positive & Brucella strain isolated \\
\hline L mutton restaurant & 9 & 5 & 5 & 1 \\
J goat farm & 2 & 2 & 2 & - \\
D butcher's & 2 & 1 & 1 & - \\
M live goat market & 1 & 0 & 0 & - \\
Relatives & 5 & 0 & 0 & 1 \\
Total & 19 & 8 & 8 & - \\
\hline
\end{tabular}

Abbreviations: RBPT=Rose Bengal plate agglutination test, SAT=Serum agglutination test.

TABLE 2. A cohort study of an outbreak of brucellosis in Hubei Province, China, 2019.

\begin{tabular}{|c|c|c|c|c|c|c|c|}
\hline \multirow{2}{*}{ Factors } & \multicolumn{2}{|c|}{ Exposed } & \multicolumn{2}{|c|}{ Unexposed } & \multicolumn{2}{|c|}{ Attack rate $(\%)$} & \multirow{2}{*}{$\mathrm{RR}(95 \% \mathrm{Cl})$} \\
\hline & Total & Cases & Total & Cases & Exposed & Unexposed & \\
\hline Slaughtering without precautions & 8 & 7 & 13 & 1 & 87.50 & 7.69 & $11.38(1.70-76.14)$ \\
\hline In slaughterhouse without precautions & 5 & 1 & 16 & 7 & 20.00 & 43.75 & $0.46(0.07-2.88)$ \\
\hline Processing frozen mutton without precautions & 8 & 0 & 13 & 8 & 0 & 61.54 & $0^{*}$ \\
\hline
\end{tabular}

Abbreviations: RR=Relative Risk.

${ }^{*} p=0.007$. 
roofed room of nine square meters with a door connecting the lobby of the restaurant. There were no windows, ventilation, or disinfection facilities, and the conditions in D butcher's were similar.

J goat farm is located beside the Yangtze River and separated from residential areas. In the off season, the farm kept about 200 self-bred goats. In November 2018, the farm bought about 100 goats from M live goat market and kept them with self-bred goats, which might have led to cross-infection. Several pregnant ewes were aborted between December 2018 and January 2019 but were still sold. There were no inspections or quarantine measures while in transit since these goats were transported through a rural road. All the goats in the farm were sold for the first time in nearly a decade in January 2019 without any inspection measure.

\section{PUBLIC HEALTH RESPONSE}

The routine control measures, including daily inspection of livestock transport on highways and annual random testing of the goats for brucella in farms during spring and autumn, failed to detect the outbreak in January 2019 when the infections were spreading so no emergency measures were taken then. During this investigation in June 2019, multiple emergency countermeasures were taken including case searching, diagnosis and treatment of patients, health education, tracing the sources of goats and mutton, and disinfecting contaminated environments. Based on this investigation, local agricultural departments and health departments took long-term joint actions including promoting health education, general surveying of goat breeding across Xianning, cracking down on illegal activities related to sick animals and animal products, etc. In addition, a special notice was issued to strengthen and adjust the eradication program of brucellosis.

\section{DISCUSSION}

This is the first outbreak of occupational brucellosis involving multiple clusters in Hubei. The investigation indicates that 2 clusters involving 5 initial cases were part of an outbreak and presented a transmission chain for a brucellosis outbreak. This report revealed that southern provinces are faced with a series of challenges and hurdles to overcome. Trans-regional goat trading and lax inspection measures might result in importation of infected goats, which were fed in the same sheepfold with local goats, and then led to cases of human brucellosis in those who slaughtered or raised these goats without protection in the process of slaughtering in January 2019. Seven cases were engaged in slaughtering and one case might have been infected by inhaling aerosols. J goat farm, L mutton restaurant and $\mathrm{D}$ butcher's all fell victims of this outbreak.

Brucellosis is spreading in humans and animals in Asian countries, and Northern China has been more heavily impacted traditionally by brucellosis than Southern China (2-3,6). Many brucellosis infections in southern provinces resulted from imported infected animals or products from Northern China (7-8), so northern provinces should strengthen brucellosis control at the source.

Neither imported goats nor the self-bred goats were found to be vaccinated, and both types of goats were all mixed together, which resulted in the spread of brucellosis among these goats. We also found that the transportation of goats was not strictly inspected. When an outbreak of animal disease occurs, the farm owners often sell all their livestock in a short time without realizing the infectiveness (4). The phenomenon of emptying stock is worthy of attention. Comprehensive control strategies should be adopted including isolated feeding of imported goats from local ones, vaccination, and inspection measures, all of which can reduce the incidence of brucellosis as evidenced by Italy (9).

Awareness and knowledge of brucellosis among occupational workers are commonly regarded as a vital aspect of human brucellosis control (10). This investigation found that local occupational workers had a low awareness of brucellosis and personal protective measures, so health education should be promoted among relevant occupational groups.

Due to the swelling demand of fresh mutton, the influx of live goats from northern provinces to the south is becoming increasingly frequent. This report reveals a series of challenges in the current eradication program of brucellosis and provides a useful reference for strengthening and adjusting the action in southern provinces.

Acknowledgments: We thank Hongjun Zhou from Xianning Municipal Center for Disease Control and Prevention (CDC), Xiaojia Song from Shiyan CDC, Xiaomin Wu from Wuhan CDC, participants from local agricultural sectors, and all other participants for their help with this investigation. 
Conflicts of interest: No conflicts of interest were reported.

\section{doi: $10.46234 / \mathrm{ccdcw} 2020.119$}

\# Corresponding authors: Yang Wu,6021975@qq.com; Xuhua Guan, 552371433@qq.com.

\footnotetext{
Nanjing Municipal Center for Disease Control and Prevention, Nanjing, China; ${ }^{2}$ Chinese Field Epidemiology Training Program (CFETP), Beijing, China; ${ }^{3}$ Hubei Provincial Center for Disease Control and Prevention, Wuhan, China; ${ }^{4}$ Chongyang Center for Disease Control and Prevention, Xianning, China; 5 Nanchang Municipal Center for Disease Control and Prevention, Nanchang, China; ${ }^{6}$ Yiling Center for Disease Control and Prevention, Yichang, China; ${ }^{7}$ Division of Infectious Disease, Key Laboratory of Surveillance and Early Warning on Infectious Disease, Chinese Center for Disease Control and Prevention, Beijing, China; ${ }^{8}$ Xianning Municipal Center for Disease Control and Prevention, Xianning, China; ${ }^{9}$ Jiayu Center for Disease Control and Prevention, Xianning, China.

\& Joint first authors.
}

Submitted: March 13, 2020; Accepted: May 14, 2020

\section{REFERENCES}

1. Joseph R, Crotty MP, Cho J, Wilson MH, Tran J, Pribble J, et al. A single-institution experience with a brucellosis outbreak in the United States. Am J Infect Control 2018;46(10):1195 - 7. http://dx.doi.org/ 10.1016/j.ajic.2018.03.022.

2. Kong WL. Brucellosis infection increasing in Southern China. Eur J Intern Med 2018;51:e16-8. http://dx.doi.org/10.1016/j.ejim.2018. 03.004 .
3. Lai SJ, Zhou H, Xiong WY, Yu HJ, Huang ZJ, Yu JX, et al. Changing epidemiology of human brucellosis, China, 1955-2014. Emerg Infect Dis 2017;23(2):184 - 94. http://dx.doi.org/10.3201/eid2302.151710.

4. Liu GP, Xing XS, Wu Y, Xiao JH, Li GM, Zhou QB, et al. Investigation on the first human brucellosis in Hubei Province, 2011. Chin J Zoonoses 2012;28(12):1258 - 60. http://dx.doi.org/10.3969/cjz. j.issn.1002-2694.2012.12.022. (In Chinese).

5. Li D, Xing XS, Liu L, Liu HH, Zhao MJ. Epidemiological characteristics of human brucellosis in Hubei, 2010-2016. Dis Surveil 2018;33(3):203 - 7. http://dx.doi.org/10.3784/j.issn.1003-9961.2018. 03.008. (In Chinese).

6. Chen JD, Ke CW, Deng XL, Jiang S, Liang WJ, Ke BX, et al. Brucellosis in Guangdong province, People's Republic of China, 20052010. Emerg Infect Dis 2013;19(5):817 - 8. http://dx.doi.org/10.3201/ eid1905.120146.

7. Zhan BD, Wang SQ, Lai SM, Lu Y, Shi XG, Cao GP, et al. Outbreak of occupational brucellosis at a pharmaceutical factory in southeast China. Zoonoses Public Health 2017;64(6):431 - 7. http://dx.doi.org/ 10.1111/zph.12322.

8. Tan ZM, Huang Y, Liu GY, Zhou WZ, Xu XL, Zhang ZB, et al. A familial cluster of human brucellosis attributable to contact with imported infected goats in Shuyang, Jiangsu Province, China, 2013. Am J Trop Med Hyg 2015;93(4):757 - 60. http://dx.doi.org/10.4269/ ajtmh.15-0149.

9. Mancini FR, Bella A, Graziani C, Marianelli C, Mughini-Gras L Pasquali P, et al. Trends of human brucellosis in Italy, 1998-2010. Epidemiol Infect 2014;142(6):1188 - 95. http://dx.doi.org/10.1017/ S0950268813002227.

10. Zeng H, Wang YM, Sun XD, Liu P, Xu QG, Huang D, et al. Status and influencing factors of farmers' private investment in the prevention and control of sheep brucellosis in China: a cross-sectional study. PLoS Negl Trop Dis 2019;13(3):e0007285. http://dx.doi.org/10.1371/ journal.pntd.0007285. 